\title{
On the application of spectral corrections to particle flux measurements
}

\section{Oosterwijk, Aleid}

2018-10-01

Oosterwijk , A , Henzing , B \& Järvi , L 2018 , ' On the application of spectral corrections to particle flux measurements ' , Environmental Science: Nano , vol. 5 , no. 10 , pp. 2315-2324 . https://doi.org/10.1039/c8en00501j

http://hdl.handle.net/10138/323381

https://doi.org/10.1039/c8en00501j

cc_by

acceptedVersion

Downloaded from Helda, University of Helsinki institutional repository.

This is an electronic reprint of the original article.

This reprint may differ from the original in pagination and typographic detail.

Please cite the original version. 


\title{
On the application of spectral corrections to particle flux measurements
}

\author{
Aleid Oosterwijk, Bas Henzing, Leena Järvi
}

9th November 2017

\begin{abstract}
To study the environmental fate of nanoparticles, reliable measurements of particle fluxes in the atmosphere are of importance. The eddy-covariance (EC) technique can be used to calculate surface fluxes. In this study, the EC technique has been applied to calculate particle fluxes in Helsinki (Finland) and Cabauw (the Netherlands). For reliable estimations for the surface fluxes, particle flux measurements need to be corrected for attenuation of the highest frequencies. This attenuation is caused by the relatively long response time of first order scalar sensors. The attenuation can be estimated using a theoretical or an empirical approach. Horst 1997 developed a simple formula to estimate the attenuation, based on the empirical approach. The empirical approach relies on the assumption of spectral similarity. In this paper the effect of the assumption of spectral similarity is investigated. It is shown that in order to apply the Horst 1997 formula a reliable estimate of the sensor response time is required. Furthermore, it is shown that in order to apply the empirical method, a fast sensor response is required. The assumption of spectral similarity seems reasonable for particle fluxes and heat fluxes. An altered assumption of spectral similarity has been applied, where similarity is assumed only after the peak frequency in the cospectrum. This assumption leads to a better estimate for the attenuation, when applied to the Helsinki data.
\end{abstract}

\section{Introduction}

The environmental fate of nanoparticles has become an area of extensive research over the past years. This increased interest is caused mainly by an intensified engineered nanoparticle production. Hence, increased effort in research on the inter- action of nanoparticles with their environment is of vital importance. One pathway that has to be studied, is the release and fate of nanoparticles into the atmosphere [John et al. 2017. Information on emission- and deposition areas, particle fluxes and their deposition velocities is of importance for modelling of the behaviour and fate of nanoparticles in the atmosphere.

The eddy covariance (EC) technique is a well-known technique, used to calculate surface fluxes of varying scalars. It has been widely used to calculate e.g. fluxes of water vapour, carbon dioxide, methane and ozone [Clifton et al., 2017 Mammarella et al. 2016. Aubinet et al. 2012]. The EC technique has also been applied for calculating particle fluxes [Pryor et al., 2008 Buzorius et al., 1998 Buzorius et al. 2000] [Fairall 1984 [Suni et al. 2003] Nilsson et al. 2001|. The post-processing of particle concentration measurements for calculating fluxes has been reported on by Aubinet et al. 1999 and Lee et al. 2006. Special care has to be taken when applying the EC method to particle fluxes. For example, particle concentrations are rarely stationary Buzorius et al. 1998, making the application of the EC method more complicated.

The eddy covariance method has also been applied to calculate size-resolved particle fluxes, as described in e.g. Nemitz et al. [2002, Buzorius et al. 2000. Also slightly adapted methods to calculate size resolved particle fluxes have been applied, by Buzorius et al. [2003], Schmidt and Klemm 2008.

Due to the relatively slow response time of particle counters, especially in measuring particle concentrations high-frequency attenuation may be significant. The resulting underestimation can be corrected using spectral corrections Buzorius et al. 2000]. In general there are two approaches for these corrections: a theoretical approach and an empirical approach, which are both described in detail in 
Aubinet et al. 2012. In the theoretical approach, the spectral corrections are based on knowledge of the effects of the measurement system and for each effect the correction is calculated separately Moore 1986. In the empirical approach, the spectral corrections are based on knowledge of what an ideal measured spectrum should look like and the difference between the measurement and this ideal spectrum is corrected.

In this paper the empirical approach is investigated. The empirical approach is based on knowledge on the shape of the power spectra and cospectra of the scalars at interest. Based on meteorological theories, they should follow a specific slope, as shown by Kaimal et al. 1972. The reference spectrum, to which the measured spectrum is compared, can be either a theoretical one or another measured spectrum. When assuming spectral similarity between sensible heat flux and particle flux, one could use the unattenuated sensible heat flux cospectrum as the reference spectrum, in order to design a transfer function for correcting the high frequencies of the particle flux cospectrum.

Spectral similarity between particle fluxes and fluxes of other scalars is based on the scalar similarity theory, according to which scalar quantities are transported within eddies of different sizes and shapes, with similar efficiency Ruppert et al. 2006 Kaimal et al., 1972. Scalar similarity between temperature, water vapour, carbon dioxide and ozone are generally assumed, and this assumption has been studied extensively De Ligne et al. 2010, [Pearson et al., 1998, Ruppert et al. 2006], showing that for water vapour in particular the assumption of scalar similarity should be made with some caution. Scalar similarity for particle concentrations has not been undisputedly proven. Ruppert et al. 2006 shows that the assumption of spectral similarity might be questionable, especially for the lower frequency part, due to the differences in sources and sinks. Even if scalar similarity between particles and other scalars can be assumed, the measured spectra might be far from the ideal spectra presented in Kaimal et al. 1972, yielding it uncertain whether the frequently-applied spectral corrections by Horst 1997. could be applied to particle flux measurements.

Spectral corrections are necessary to obtain reliable flux estimates with the eddy covariance method. In this paper we investigate the effect of the assumption of spectral similarity on the high-frequency spectral corrections that can be applied to particle fluxes. The transfer function based on comparison with measurements of other scalars will be compared to the transfer function of Horst 1997, which is based on a theoretical approach. Section 2 explains the theory of spectral corrections based on empirical methods, and Section 3 describes the measurement set-ups and post-processing steps. In Section 4, the results are presented for two measurement sites. The main conclusions and discussion follow in the last section.

\section{Theory}

The co-spectral transfer function for spectral corrections can be determined as Aubinet et al. 2012

$$
T F(f)=\frac{N_{T}}{N_{s}} \frac{C_{w s}(f)}{C_{w T}(f)},
$$

where $f$ is the frequency, $N_{T}$ and $N_{s}$ are the normalization factors for the sensible heat flux cospectrum and the cospectrum of another scalar, and $C_{w s}$ and $C_{w T}$ are the scalar flux cospectrum and heat flux cospectrum. In our analysis, the scalar of interest is the particle flux.

The normalization factors $N_{T}$ and $N_{s}$ of the cospectra can be determined by integrating the cospectra over a suitable frequency range:

$$
\frac{N_{T}}{N_{s}}=\frac{\int_{f_{1}}^{f_{2}} C_{w T}(f) d f}{\int_{f_{1}}^{f_{2}} C_{w s}(f) d f},
$$

where $f_{2}$ and $f_{1}$ have to be determined. The integration should be performed only on the part of the spectrum where spectral similarity can be assumed. Regions where differences between the heat flux spectrum and particle flux spectrum are expected should be excluded from the normalisation. Because there is attenuation at the high frequency end of the particle spectrum, one should not normalize with the total flux, as this would include that the attenuated part. Therefore, the spectrum is integrated up to a certain frequency $f_{2}$, below which negligible attenuation is assumed, as explained in more detail in Aubinet et al. 2012.

In general, it is assumed that $f_{1}=0$, i.e. the lowfrequency part is suitable for normalisaton. However, comparing the temperature cospectrum with the particle cospectrum can only be done under the assumption of spectral similarity. If this cannot be 
assumed, the ratio of the spectra cannot be used to determine the transfer function. Following the conclusions of Ruppert et al. 2006], it is suspected that poor scalar similarity might be found at low frequencies. Therefore, when spectral similarity is not assumed, the normalisation of the spectra is based on an interval between $f_{1}=f_{m}$ and $f_{2}$, where $f_{m}$ is the frequency at which the cospectrum is at its maximum. The cospectra figures in this paper are shown versus normalised frequency, defined as

$$
n=\frac{z}{\bar{u}} f
$$

where $z$ is the height above the canopy of the measurement and $\bar{u}$ is the mean wind speed. Therefore, the integration of the spectra is done using $n_{m}=\frac{z}{\bar{u}} f_{m}$ and $n_{2}=\frac{z}{\bar{u}} f_{2}$, which are the normalised frequency at which the cospectrum is at its maximum and normalised upper bound frequency respectively.

The approximate position of the peak has been determined based on theoretical considerations Horst, 1997. It can be expressed in normalised frequency $n$, as

$$
n_{m}= \begin{cases}0.085 & \text { if } z / L \leq 0 \\ 2-1.915 /(1+0.5 z / L) & \text { if } z / L>0\end{cases}
$$

where $n_{m}$ is the normalised frequency at which the (co)spectrum is at its maximum and $L$ is the Obhukov length, approximated by $L=-\frac{u_{*}^{3} T}{k g Q}$, where $Q$ is the temperature flux, $T$ is the temperature in Kelvin, $k$ is the von Kármán constant, $g$ is the gravitational acceleration and $u_{*}=\left({\overline{u^{\prime} v^{\prime}}}^{2}+{\overline{v^{\prime} w^{\prime}}}^{2}\right)^{1 / 4}$ is the friction velocity. The position of the peak in the idealised model in Horst 1997 is the best a priori estimate of the position of the peak and is therefore used to determine the integration limit $f_{2}$ in Eq. 2, even though the position of the peak in the measured, non-ideal spectrum might be positioned differently.

The value of $n_{2}$ can be estimated using the transfer function from Horst 1997,

$$
T F(f)=\frac{1}{1+(2 \pi f \tau)^{2}},
$$

where $\tau$ is the characteric time of the sensor response. This is an estimate of the attenuation based only on the time constant. Therefore, it should be used with caution, because the Horst transfer function will be compared to the empirically determined transfer function. The empirically determined transfer function also takes into account attenuation caused by other parts of the measurement setup, e.g. the tube. Finding large differences between the empirical transfer function and Horst transfer function would form a basis for questioning the reliability of $n_{2}$. To determine $n_{2}$, it is required to define a threshold $A$, which is the amount of signal that should be retained. E.g., when $A=0.9$, this means that only frequencies for which there is a maximum loss by attenuation of $10 \%$ are included in the normalisation. Using $A$, one can determine $n_{2}$ as

$$
n_{2}=\frac{z}{\bar{u}} f_{t}=\frac{z}{\bar{u}} \frac{\sqrt{1 / A-1}}{2 \pi \tau} .
$$

The attenuation factor $a$ can be determined as

$$
a=\frac{\int T F(f) C_{w s}(f) d f}{\int C_{w s}(f) d f} .
$$

Horst 1997 proposes a formula to determine the attenuation without integrating the cospectrum:

$$
a=\frac{1}{1+\left(2 \pi f_{m} \tau\right)^{\alpha}},
$$

where $a$ is proposed to be either 1 or $7 / 8$.

\section{Method}

\subsection{Measurement setup}

Flux measurements in this study are performed at two different sites. The SMEARIII (Station for Measuring Ecosystem-Atmosphere Relations) satellite site Hotel Torni $(60.1678 \mathrm{E}, 24.9387 \mathrm{~N})$ is located in densely built Helsinki city centre with built-up fraction of $78 \%$ Karsisto et al. 2015]. The measurements are made on top of a tower of a building where 2.3 meters high mast has been installed resulting a total height of $60 \mathrm{~m}$ above the ground level. The mean building height of the surroundings is 24.1 meters and thus the measurements are conducted at sufficient height above the blending height. The current measurement setup consists of a 3D ultrasonic anemometer (Metek USA-1, Metek $\mathrm{GmbH}$ ) and a TSI Condensation Particle Counter (CPC model 3010). The inlet of the tube leading 
to the $\mathrm{CPC}$ is located $0.3 \mathrm{~m}$ below the anemometer centre and the tube consists of two parts: $7.6 \mathrm{~m}$ long stainless steel tube and $1.0 \mathrm{~m}$ long PVS tube with inner diameters of 8 and $4 \mathrm{~mm}$, respectively. The flow rate in the tube is $12 \mathrm{l} \mathrm{min}^{-1}$ and the flow

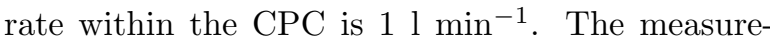
ment period between 1 and 14 November 2015 is used in this study.

The Cabauw Experimental Site for Atmospheric Research (CESAR) Observatory ${ }^{1}$ is located in the western part of the Netherlands $\left(51.971^{\circ} \mathrm{N}\right.$, $4.927^{\circ} \mathrm{E}$ ) in a polder $0.7 \mathrm{~m}$ below average sea level. The nearby area is dominated by flat agricultural grasslands with relatively little industry and households. In contrast, in the wider surrounding area at distances of 15 - 50 kilometers away, more than 10 million people live and work in one of the most densely populated areas in Europe. The CESAR Observatory includes a 212 meter tall tower specifically built for meteorological research and air pollution studies.

The eddy covariance system that measures turbulent fluxes of aerosol is placed in the tower at 68 meter above ground. The system consists of a Gill Instruments 3D ultrasonic anemometer (WindMaster Part 1590-PK-020) and a TSI Condensation Particle Counter (CPC 3775). The first instrument measures the three wind speed components $\mathrm{u}, \mathrm{v}$ and $\mathrm{w}$ along $\mathrm{x}, \mathrm{y}, \mathrm{z}$ axes, the wind direction and the sonic temperature. Aerosol particle number concentration is measured with a CPC that detects particles larger than $4 \mathrm{~nm}$ in diameter. The d air, drawn $15 \mathrm{~cm}$ away from the anemometer measurement volume, is transported to the $\mathrm{CPC}$ in a stainless steel inlet tube of $2.10 \mathrm{~m}$ length and 0.25 inch outer diameter. The delay time between entering the tube and final counting was determined experimentally at $10.5 \pm 0.5 \mathrm{~s}$ by single pulse exposure to aerosol spray. CPC and anemometer were connected to the same computer with a data acquisition frequency fixed at $10 \mathrm{~Hz}$. Measurements are performed between April 29, 2016 and February 24, 2017.

The delay time between entering the inlet tube and final recording was determined theoretically and experimentally. The laboratory experiment consisted of multiple repetitions of instantaneously removing a High Efficiency Particulate Air (HEPA) filter after being attached to the $\mathrm{CPC}$ via the sampling line for

\footnotetext{
$\sqrt[1]{\text { http://www.cesar-observatory.nl }}$
}

sufficiently long time. Doing so, after some death time, with still zero concentration, the CPC starts to record increasing concentrations until the lab-air concentrations are reached. The timeseries of all repetitions were aligned so that the exact time of removal of the HEPA were synchronized at $t=0 \mathrm{~s}$. The time constants of the CPC has been determined by $c(t)=e^{\frac{t-t_{0}}{\tau}}$, where $t_{0}$ marks the first response to lab-air and tau is the exponential time scale of growth. For our instrumental set-up, we found $t_{0}=9.7$ and $\tau=2 \pm 0.1 \mathrm{~s}$. Removal of the HEPA is sensitive to systematic errors by human action and possibly biases to low. To calculate the travel time between inlet and optical sensing, we measured length and internal diameter of all individual external and internal parts, such as e.g. connecting tubes, saturator wick and condenser. For a laminar flow profile the average travel time was determined at 10.1s. When this value is to be compared with the experiments an additional 1.3 second is to be added since this is the time between detection and final recording, i.e. when manufacturer software is used instead of pulse counting. Moreover, the calculations are based on the average velocity of the parabolic-shaped laminar flow. Centrally transported particles therefore reach the detector space much quicker. Matching the theoretical and experiment time constants is not our goal, hence we adopt another technique to determine the relevant time constants, described in the post-processing section, and used the range of values hereabove for sanity check.

The time constants of both CPCs have also been determined by fitting $c(t)=e^{\frac{t-t_{0}}{\tau}}$ to the concentration response curves in their respective manuals. This yields a time constant for the TSI775 of $\tau \approx 0.65$ and a time constant for the TSI3010 of $\tau \approx 0.85$. This compares well to the time constant of TSI3010 as determined by Buzorius 2001 of $\tau=0.83$. However, the time constant of the TSI3010 that has been used in the Helsinki set-up has been tested in the lab by Enroth et al., 2017, which yielded $\tau=0.73$. In this study we will use $\tau=0.65$ for the Cabauw data set and $\tau=0.73$ for the Helsinki data set.

\subsection{Post-processing}

To apply the eddy covariance method, postprocessing of the concentration and wind measurements is necessary. To the wind speed vector a co- 
ordinate rotation is applied along two axes, using the method first described by Tanner and Thurtell [1969]. Lag time corrections have been applied to the concentration measurement data. This is done using a prescribed time-lag that has been determined by maximizing the correlation between the vertical wind speed and the concentration measurements, averaged over a longer period of time. This lag time was confirmed experimentally. The vertical wind speed and particle concentration measurements have been linearly detrended per 30-minute block and the corresponding high-pass filter spectral correction was applied. Rannik and Vesala. 1999 After applying this detrending method a stationarity test is applied, as described in Aubinet et al. 1999. In this test the flux of a 30-minute block is calculated for six 5-minute blocks. The average of these fluxes should not deviate more than a preset percentage from the 30-minute block flux value. The threshold for discarding the data samples has been set to $30 \%$. Also wind-direction filtering has been applied. For the Helsinki data set all data between 50 and 185 degrees has been discarded, for the Cabauw data set all data between 90 and 270 degrees has been discarded. The transfer function should be determined by averaging a significant amount of spectra. For this purpose, a suitable period is to be selected, with sunny, stationary conditions Aubinet et al. 2012. Before calculating the average, the spectra are translated in frequency space - in such a way that their maxima $n_{m}$ overlap. The stationary data is selected and logarithmically averaged. The scaling of the spectra is done by integrating the area below the curve, using the factors $N_{T}$ and $N_{s}$. This is done over the entire normalised frequency range, using an upper bound given by Equation 6 and using both an upper bound and lower bound, given by Eqs. 4 and 6 . where $A=0.98$ was chosen.

\section{Results}

The effect of the assumption on spectral similarity on the empirical method for attenuation has been investigated, with the aim to determine whether spectral similarity should be assumed when determining the transfer function, and to compare the empirical method with the Horst attenuation formula. Using the different scalings $N_{s}$ and $N_{T}$, the averaged cospectra of the sensible heat flux and particle

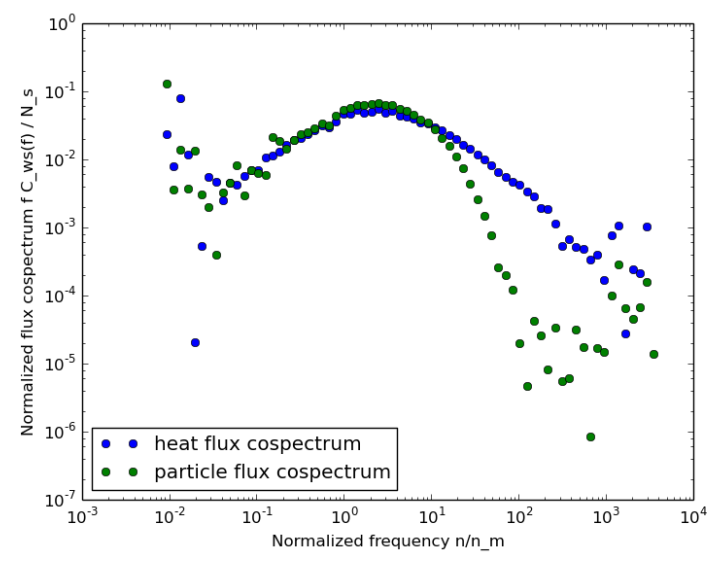

Figure 1: Heat flux cospectrum and particle flux cospectrum of Helsinki dataset, scaled with the total area under the curve.

flux have been normalised with respect to one another. The results are visualised in Figures 1, 2, and 3. The maximum of both the heat flux cospectrum and the particle flux cospectrum is not positioned at $n_{m}$ - the theoretical position of the maximum - but is shifted towards higher frequencies. This shows that the theoretical peak position might not be a good estimate of the true peak position. In other studies, a site-dependent formula for the peak is determined from the measurement data, showing that indeed the position of the peak depends on site-dependent circumstances. [Järvi et al. 2009 Rannik et al., 2004

Figure 3 reveals more overlap in the area between $n_{m}$ and $n_{2}$ in comparison to Figures 1 and 2 . When the low-frequency domain is also included in the normalisation, the particle flux cospectrum is larger than the heat flux cospectrum near the peak. Hence the particle flux would be underestimated if the lowfrequency domain is included in the normalisation. Using the different scalings as applied in Figures 1. 2 and 3 , the transfer function for the high frequency corrections has been determined. The resulting empirical transfer function is shown in Figure 4, together with the transfer function of Horst 1997]. Figure 4 displays both the $\tau$ value of the CPC and the $\tau$ value resulting from fitting the Horst function to the empirical transfer function. Excluding the high-frequency part of the domain does not yield a different transfer function in comparison to when all frequencies are included. An explanation for this 


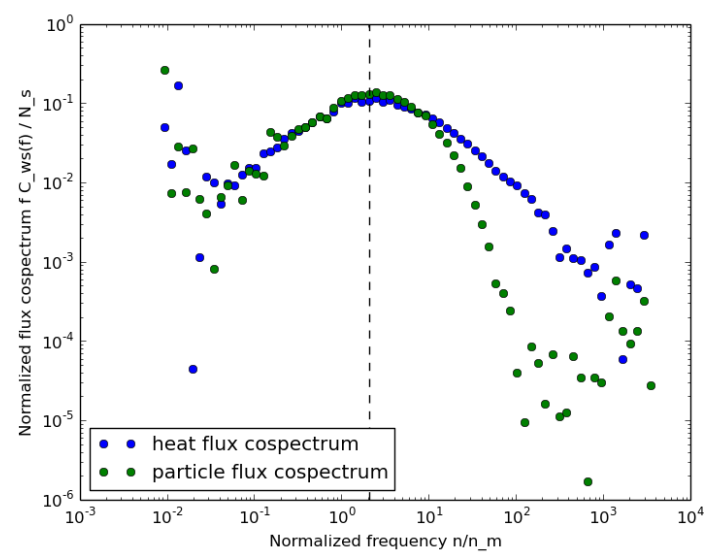

Figure 2: Heat flux cospectrum and particle flux cospectrum of Helsinki dataset, scaled with the area under the curve up to $n_{2}$ (vertical line).

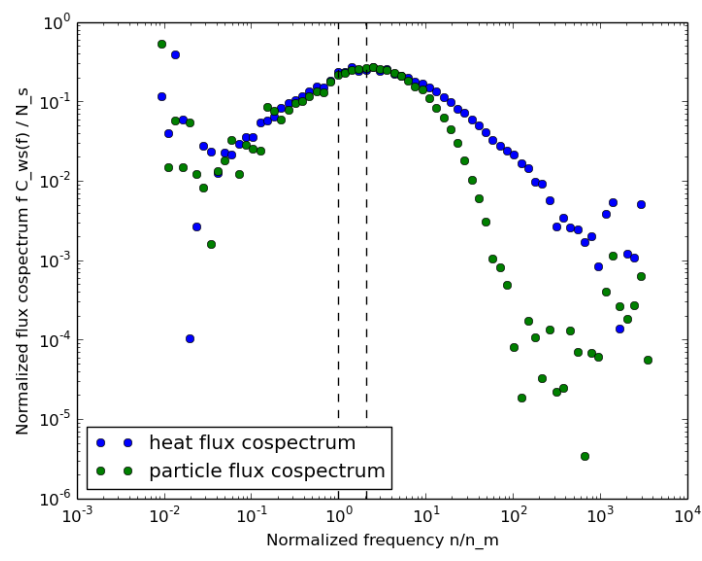

Figure 3: Heat flux cospectrum and particle flux cospectrum of Helsinki dataset, scaled with the area under the curve between $n_{m}$ (left vertical line) and $n_{2}$ (right vertical line).

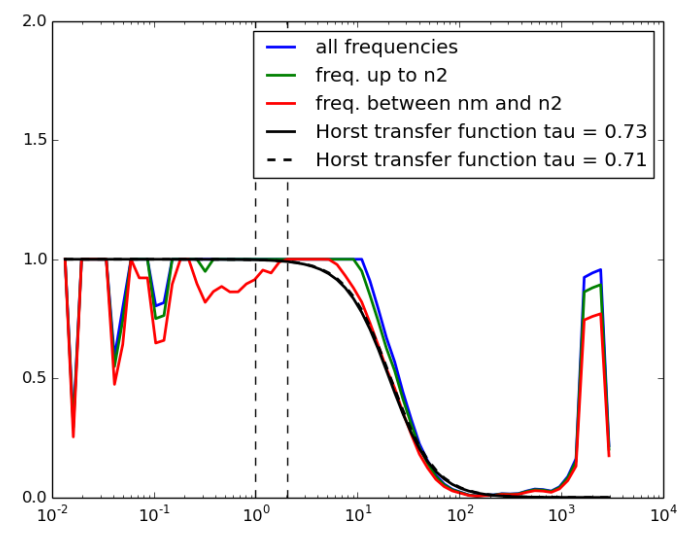

Figure 4: Transfer function of Helsinki dataset, empirically determined after scaling with the total area under the curve (blue), the area under the curve up to $n_{2}$ (right vertical line) (green), and the area under the curve between $n_{m}$ (left vertical line) and $n_{2}$ (red). Transfer function according to Horst Horst, 1997 using $\tau=0.73$ and fitted to the red curve, yielding $\tau=0.71$.

is that the position of the peak is shifted towards higher frequencies. Figure 2 reveals that the underestimation of the highest frequencies of the particle flux cospectrum is compensated by an overestimation at the peak. Note that the peak is also excluded when excluding frequencies above $n_{2}$.

Excluding the low-frequency part and the highfrequency part of the domain decreases the transfer function. The shape is similar to the transfer function of Horst 1997, except for the low- and highfrequency range. Fitting the Horst 1997 transfer function to the empirical transfer function using the smallest scaling area yields $\tau=0.71$, which is a slightly faster response time than that of the CPC, which was $\tau \approx 0.73$. We would expect a slower response time of the set-up in total, as compared to the response time of the CPC alone. An explanation for this could be a strong attenuation of the heat flux itself, which would lead to an underestimation of the particle flux attenuation. Correcting the heat flux for attenuation, using the theoretical approach as described in Aubinet et al. 1999 to estimate the attenuation, yields a value of $\tau=0.80$, which is higher than the CPC response time.

The same procedure has been applied to the Cabauw data. The results are shown in Figures 5. 
6, 7 and 8 . These figures portray that the peak of the heat flux cospectrum is positioned at $n_{m}$, as opposed to the peak of the heat flux cospectrum of the Helsinki dataset, which was shifted towards higher frequencies. Inspecting the shape of the particle flux cospectrum reveals a shift in the position of its peak. This shift may be caused by the much stronger attenuation. Because frequencies around $n_{m}$ are attenuated quite strongly for the particle flux cospectrum, the peak itself is attenuated, shifting the observed peak towards lower frequencies. Consequently, $n_{2}$ is not a suitable estimate for the upper bound of the normalisation area. The upper bound should be lower, even lower than $n_{m}$.

When the high frequency part of the domain is excluded in the scaling, this results in lower values for the transfer function. However, when excluding both the low-frequency part and the high-frequency part, the opposite occurs and the transfer function has higher values. This is supported by the considerable overlap of the cospectra, as shown in Figure 6. This result can be explained by the attenuation of the particle flux peak, making the area between $n_{m}$ and $n_{2}$ unrealiable for normalisation.

Fitting the Horst [1997] transfer function to the empirical transfer function using the smallest scaling area yields $\tau=1.78$, which is a longer response time than that of the CPC. However, from Figures 5,6 and 7 can be concluded that the actual value of $\tau$ should be even higher. This supports earlier findings in this research, concerning the strong attenuation present at the peak frequency of the particle flux spectrum. An explanation for this is the additional attenuation that is present in the tube-system towards the CPC. The determined lag-time is approximately 10 seconds, which means that before the air enters the CPC there might also be a significant amount of mixing and additional attenuation. Especially as the flow-rate is $0.3 \mathrm{lpm}$, yielding a Reynolds number within the laminar flow region. The Helsinki data set did not show this effect and has a lag time of approximately 5 seconds and a Reynolds number in the turbulent flow region.

Table 1 lists the attenuation factors as determined by all methods described in this paper. The strongest attenuation corresponds to $a=0.81$, which is a loss of $19 \%$ of the flux. This means none of our results yield an attenuation of high magnitude. However, the different methods yield quite different results. The different estimates of $\tau$ for the Cabauw data influence the estimated attenu-

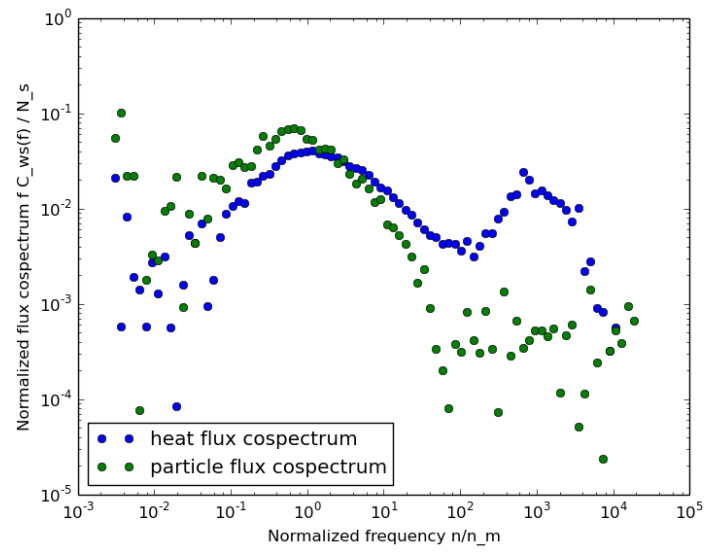

Figure 5: Heat flux cospectrum and particle flux cospectrum of Cabauw dataset, scaled with the total area under the curve.

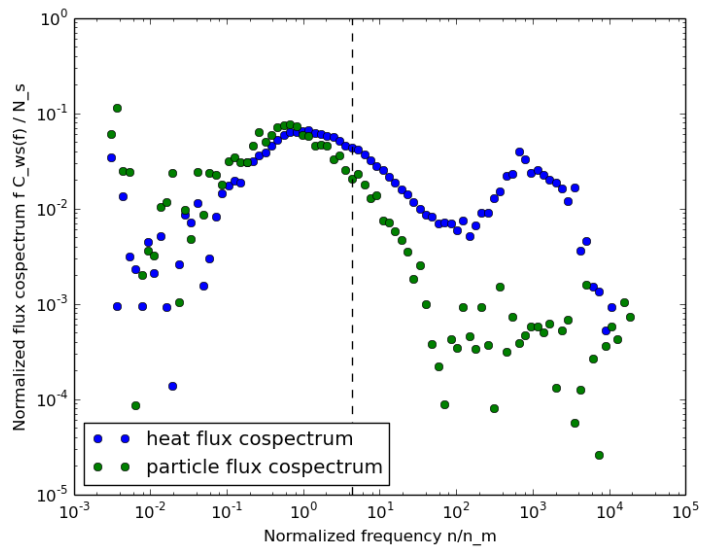

Figure 6: Heat flux cospectrum and particle flux cospectrum of Cabauw dataset, scaled with the area under the curve up to $n_{2}$ (vertical line). 


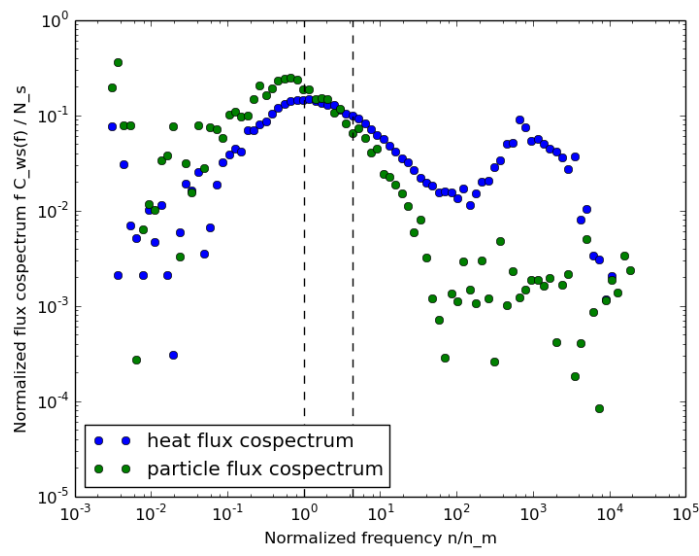

Figure 7: Heat flux cospectrum and particle flux cospectrum of Cabauw dataset, scaled with the area under the curve between $n_{m}$ (left vertical line) and $n_{2}$ (right vertical line).

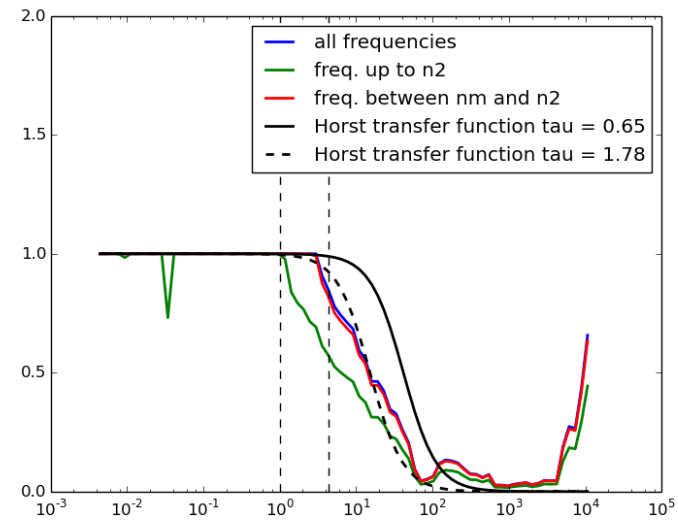

Figure 8: Transfer function of Cabauw dataset, empirically determined after scaling with the total area under the curve (blue), the area under the curve up to $n_{2}$ (right vertical line) (green), and the area under the curve between $n_{m}$ (left vertical line) and $n_{2}$ (red). Transfer function according to Horst Horst 1997 using $\tau=0.65$ and fitted to the red curve, yielding $\tau=1.78$.

\begin{tabular}{l|ll} 
& Helsinki & Cabauw \\
\hline Empirical TF & & \\
\hline All frequencies & 0.98 & 0.97 \\
Freq. up to $f_{2}$ & 0.97 & 0.90 \\
Freq. between $f_{1}$ and $f_{2}$ & 0.92 & 0.96 \\
\hline Horst TF & & \\
\hline Theoretical $\tau$ & 0.95 & 0.99 \\
Fitted $\tau$ & 0.95 & 0.97 \\
\hline Horst attenuation & & \\
\hline Theoretical $\tau, \alpha=1$ & 0.95 & 0.94 \\
Fitted $\tau, \alpha=1$ & 0.95 & 0.87 \\
Theoretical $\tau, \alpha=7 / 8$ & 0.93 & 0.93 \\
Fitted $\tau, \alpha=7 / 8$ & 0.93 & 0.86
\end{tabular}

Table 1: Attenuation factors $a$ for the Helsinki and Cabauw data set, as determined by applying the empirically determined transfer function in Eq. 7 (Empirical TF), by applying the Horst transfer function, using the theoretical and fitted $\tau$-value in Eq. 7 (Horst TF) and by using the Horst attenuation formula (Eq. 8), using the theoretical and fitted $\tau$-value and the $\alpha$-values proposed by Horst. (Horst attenuation)

ation considerably. Looking at the Cabauw results, applying the Horst transfer function in Eq. 7 to determine the attenuation yields considerably higher values for $a$ than applying the Horst attenation formula of Eq. 8

\section{Discussion}

The effect of the assumption of spectral similarity on the estimation of the attenuation of particle fluxes has been investigated, and the empirical method to estimate the attenuated loss was compared against the Horst attenuation formula. The attenuation at the Cabauw site is much stronger than at the Helsinki site, even though the CPC used at the Cabauw site has a slightly smaller response time. This effect could be caused by a difference in measurement set-up. The lag times of the setups differ significantly and the flow is laminar for the Cabauw set-up and turbulent for the Helsinki set-up.

A fast response time is essential for applying the empirical method. When the response time is too low, the area viable for normalisation of the particle flux cospectrum with respect to the heat flux cospec- 
trum is too small to yield a reliable result. Therefore, a fast particle counter is a requirement to apply the empirical method.

The shape of the empirical transfer function and Horst transfer function are in accordance. The Horst transfer function is therefore a good approximation, but only if the $\tau$-value is reliable. When the estimate of $\tau$ deviates too strongly from the real value, the resulting attenuation factor will deviate too strongly for reliable results. A reliable estimate of $\tau$ is therefore a requirement to apply the Horst attenuation formula.

The Horst attenuation formula is more straightforward to apply, in comparison to the Horst transfer function. It seems that applying the Horst transfer function to the actual spectra does not yield better results than using the Horst attenuation formula, based on our findings. Therefore using the Horst attenuation factor seems preferable over the Horst transfer function.

It is difficult to draw final conclusions on whether to apply the empirical method or the Horst attenuation formula. The applicability of the methods depends on the estimate of $\tau$ and the estimate of the integration bounds $f_{1}$ and $f_{2}$, and the use of a fast particle counter. Also it is essential to have a good estimate of the attenuation of the heat flux itself. Both methods depend on spectral similarity. Considering the heat flux cospectra and particle flux cospectra of the Helsinki and Cabauw data, the assumption of spectral similarity between heat fluxes and particle fluxes seems reasonable.

In order to determine definitely which method to estimate the attenuation yields the best results when applied to particle fluxes, further research is necessary. The conclusion that the attenuation is stronger at the Cabauw site than at the Helsinki site, even though CPC that has been used has a faster response time, shows the need for a turbulent flow in the measurement-system.

\section{Acknowledgement}

TODO: What should I write here? NanoFase, Actris

\section{References}

Marc Aubinet, Achim Grelle, Andreas Ibrom, Ü Rannik, J Moncrieff, Th Foken, AS Kowalski,
PH Martin, P Berbigier, Ch Bernhofer, et al. Estimates of the annual net carbon and water exchange of forests: the euroflux methodology. $A d$ vances in ecological research, 30:113-175, 1999.

Marc Aubinet, Timo Vesala, and Dario Papale. Eddy covariance: a practical guide to measurement and data analysis. Springer Science \& Business Media, 2012.

G Buzorius, Ü Rannik, JM Mäkelä, P Keronen, $\mathrm{T}$ Vesala, and M Kulmala. Vertical aerosol fluxes measured by the eddy covariance method and deposition of nucleation mode particles above a scots pine forest in southern finland. Journal of Geophysical Research: Atmospheres, 105(D15): 19905-19916, 2000.

G Buzorius, Ü Rannik, ED Nilsson, T Vesala, and M Kulmala. Analysis of measurement techniques to determine dry deposition velocities of aerosol particles with diameters less than $100 \mathrm{~nm}$. Journal of Aerosol Science, 34(6):747-764, 2003.

Gintautas Buzorius. Cut-off sizes and time constants of the cpc tsi 3010 operating at 1 ? $3 \mathrm{lpm}$ flow rates. Aerosol Science \& Technology, 35(1): 577-585, 2001.

Gintautas Buzorius, Üllar Rannik, Jyrki M Mäkelä, Timo Vesala, and Markku Kulmala. Vertical aerosol particle fluxes measured by eddy covariance technique using condensational particle counter. Journal of Aerosol Science, 29(1-2):157171, 1998.

O. E. Clifton, A. M. Fiore, J. W. Munger, S. Malyshev, L. W. Horowitz, E. Shevliakova, F. Paulot, L. T. Murray, and K. L. Griffin. Interannual variability in ozone removal by a temperate deciduous forest. Geophysical Research Letters, 44:542-552, 2017. doi: 10.1002/ 2016GL070923.

Anne De Ligne, Bernard Heinesch, and Marc Aubinet. New transfer functions for correcting turbulent water vapour fluxes. Boundary-layer meteorology, 137(2):205-221, 2010.

Joonas Enroth, Juha Kangasluoma, Frans Korhonen, Susanne Hering, David Picard, Greg Lewis, Michel Attoui, and Tuukka Petj. On the time response determination of condensation 
particle counters. Submitted to Aerosol Science and Technology, 2017.

CW Fairall. Interpretation of eddy-correlation measurements of particulate deposition and aerosol flux. Atmospheric Environment (1967), 18 (7):1329-1337, 1984.

TW Horst. A simple formula for attenuation of eddy fluxes measured with first-order-response scalar sensors. Boundary-Layer Meteorology, 82(2):219 233, 1997.

Leena Järvi, Ivan Mammarella, Werner Eugster, Andreas Ibrom, Erkki Siivola, Ebba Dellwik, Petri Keronen, George Burba, and Timo Vesala. Comparison of net co2 fluxes measured with open-and closed-path infrared gas analyzers in an urban complex environment. Boreal Environment Research, 14:499-514, 2009.

Astrid C John, Miriam Küpper, Astrid MM Manders-Groot, Bruno Debray, Jean-Marc Lacome, and Thomas AJ Kuhlbusch. Emissions and possible environmental implication of engineered nanomaterials (enms) in the atmosphere. Atmosphere, 8(5):84, 2017.

JC Kaimal, JCj Wyngaard, Y Izumi, and OR Coté. Spectral characteristics of surface-layer turbulence. Quarterly Journal of the Royal Meteorological Society, 98(417):563-589, 1972.

Petteri Karsisto, Carl Fortelius, Matthias Demuzere, C.S.B. Grimmond, Keith Oleson, R. Kouznetsov, Valery Masson, and Leena Järvi. Seasonal surface urban energy balance and wintertime stability simulated using three landsurface models in the high-latitude city helsinki. Q. J. R. Meteorol. Soc., 142(694), 2015. doi: $10.1002 /$ qj. 2659.

Xuhui Lee, William Massman, and Beverly Law. Handbook of micrometeorology: a guide for surface flux measurement and analysis, volume 29. Springer Science \& Business Media, 2006.

Ivan Mammarella, Olli Peltola, Annika Nordbo, Leena Järvi, and Üllar Rannik. Quantifying the uncertainty of eddy covariance fluxes due to the use of different software packages and combinations of processing steps in two contrasting ecosystems. Atmos. Meas. Tech., 9:4915-4933, 2016. doi: 10.5194/amt-9-4915-2016.
CJ Moore. Frequency response corrections for eddy correlation systems. Boundary-Layer Meteorology, 37(1-2):17-35, 1986.

Eiko Nemitz, Martin W Gallagher, Jan H Duyzer, and David Fowler. Micrometeorological measurements of particle deposition velocities to moorland vegetation. Quarterly Journal of the Royal Meteorological Society, 128(585):22812300, 2002.

ED Nilsson, Ü Rannik, Eric Swietlicki, Caroline Leck, Pasi P Aalto, Jingchuan Zhou, and M Norman. Turbulent aerosol fluxes over the arctic ocean: 2. wind-driven sources from the sea. Journal of Geophysical Research: Atmospheres, 106(D23):32139-32154, 2001.

RJ Pearson, SP Oncley, and AC Delany. A scalar similarity study based on surface layer ozone measurements over cotton during the california ozone deposition experiment. Journal of Geophysical Research: Atmospheres, 103(D15): 18919-18926, 1998.

SC Pryor, M Gallagher, H Sievering, Sören Ejling Larsen, Rebecca Jane Barthelmie, F Birsan, E Nemitz, J Rinne, M Kulmala, T Grönholm, et al. A review of measurement and modelling results of particle atmosphere-surface exchange. Tellus B, 60(1):42-75, 2008.

Üllar Rannik and Timo Vesala. Autoregressive filtering versus linear detrending in estimation of fluxes by the eddy covariance method. BoundaryLayer Meteorology, 91(2):259-280, 1999.

Üllar Rannik, Petri Keronen, Pertti Hari, and Timo Vesala. Estimation of forest-atmosphere co 2 exchange by eddy covariance and profile techniques. Agricultural and Forest Meteorology, 126(1):141155, 2004.

Johannes Ruppert, Christoph Thomas, and Thomas Foken. Scalar similarity for relaxed eddy accumulation methods. Boundary-Layer Meteorology, 120(1):39-63, 2006.

A Schmidt and O Klemm. Direct determination of highly size-resolved turbulent particle fluxes with the disjunct eddy covariance method and a 12-stage electrical low pressure impactor. Atmospheric Chemistry and Physics, 8(24):7405-7417, 2008. 
Tanja Suni, Janne Rinne, Anni Reissell, Nuria Altimir, Petri Keronen, U Rannik, MD Maso, Markku Kulmala, and Timo Vesala. Long-term measurements of surface fluxes above a scots pine forest in hyytiala, southern finland, 19962001. Boreal Environment Research, 8(4):287$302,2003$.

Champ B Tanner and George W Thurtell. Anemoclinometer measurements of reynolds stress and heat transport in the atmospheric surface layer. Technical report, WISCONSIN UNIVMADISON DEPT OF SOIL SCIENCE, 1969. 